\title{
THE FATE OF ULTRALUMINOUS MERGERS
}

\author{
A.C. BAKER AND D.L. CLEMENTS \\ Service d'Astrophysique, C.E.A. Saclay, Orme de Merisiers \\ Bât. 709, F91191 Gif-sur-Yvette CEDEX, France.
}

Galaxy formation is a diverse range of ongoing processes. Numerical simulations suggest that disk galaxies in collision pass through a massive burst of star formation, and produce 'elliptical-like' remnants similar to bone fide elliptical galaxies. The observed relative numbers of merging systems and elliptical galaxies are consistent with this picture (Toomre 1977). We here investigate further by studying the distribution of old stars in a sample of merging galaxies : the ultraluminous IR galaxies (ULIRGs). We selected ten ULIRGs from the literature (Clements \& Baker 1996; Leech et al. 1994; Zhenglong et al. 1991; Melnick \& Mirabel 1990) by two criteria: proximity (redshifts $z<0.15$ ), for good spatial resolution; and confirmed signs of merging. We obtained deep K-band images $\left(t_{\text {int }} \sim 1000-2700\right.$ s $)$ in good seeing using MAGIC on the Calar Alto $3.5 \mathrm{~m}$ telescope. Our data have a field-of-view roughly equivalent to $10-20$ effective radii for the galaxies. We have fitted analytic surface brightness profiles to the data for the exponential disk and the de Vaucouleurs $r^{1 / 4}$ elliptical descriptions. We strongly favour the elliptical-like description in 8 out of 10 cases, supporting the picture that collision and merger of classical spiral galaxies can produce classical elliptical galaxies, through tidal disruption, violent star formation accompanied by prodigious infrared emission, and gravitational relaxation. These data can also constrain the properties of the ULIRG (double) nuclei and hence the lifetime of the ULIRG phase (Baker \& Clements 1998).

\section{References}

Baker, A.C., \& Clements, D.L. 1998, in preparation

Clements, D.L. , \& Baker, A.C. 1996, $A \& A$, 314, L5

Leech, K.J. et al. 1994, MNRAS, 267, 253

Melnick, J. \& Mirabel, I.F. 1990, $A \& A$, 231, L19

Toomre, A. 1977 in The Evolution of Galaxies and Stellar Populations, ed. B.M. Tinsley,

R. B. Larson, (New Haven:Yale U.Obs.), p.401,

Zhenglong, Z., et al. 1991, MNRAS, 252, 593 